\title{
Pervasive technologies and assistive environments: cognitive systems for assistive environments: special issue of PETRA 2010 and 2011 conferences
}

\author{
Ilias Maglogiannis • Fillia Makedon • \\ Grammati Pantziou $\cdot$ Margrit Betke
}

Published online: 19 July 2013

(C) Springer-Verlag Berlin Heidelberg 2013

The use of computing technology to improve everyday life continues at an exponential rate. Computer technology is becoming an integral part of environments addressing important social and healthcare issues [1-5]. On the other hand, improving the quality of living environments to enable a person to reach his or her maximum potential becomes particularly important in an increasingly crowded and complex world in which inclusiveness and connectivity with the rest of the world are critical. Cognitive systems for assistive environments address the fact that people increasingly rely on technology in their own homes $[6,7]$ as well as in work environments. This special issue addresses aspects of intelligent and context-aware computer technology that is designed to assist persons with special needs. It focuses on the use of devices (e.g., different types of sensors), tools and methods that can monitor, assess and facilitate the functionality of a person's everyday routine. By using, for example, camera-based sensors, touch sensors, heat sensors, in combination with special databases, personal area networks, machine learning and other computing methods, the aim is to achieve

\section{Maglogiannis $(\square)$}

Department of Digital Systems, University of Piraeus,

80, Karaoli and Dimitriou Str, 18534 Piraeus, Greece

e-mail: imaglo@gmail.com; imaglo@unipi.gr

\section{F. Makedon}

Heracleia Human Centered Computing Lab, University of Texas at Arlington, Arlington, TX, USA

G. Pantziou

Department of Informatics, TEI of Athens, Athens, Greece

\section{Betke}

Department of Computer Science, Boston University, Boston, MA, USA ubiquitous connectivity, distributed intelligence and interactivity through adaptive and easy-to-use interfaces.

This special issue comes after the successful organization of the 3rd and 4th International Conferences on "PErvasive Technologies Related to Assistive Environments_PETRA 2010 and 2011," which were held in Corfu and Herakleion, Greece (http://www.petrae.org/). This UAIS special issue is focused on research related to the design, development, evaluation and use of cognitive and context-aware systems for assistive environments, taking also into account the target audience of the UAIS Journal. As a result, nine (9) papers describing systems, algorithms and methodologies from the area of cognitive pervasive systems comprise the special issue.

More specifically, Kwan et al. [8] in their paper propose interaction techniques that can be used with mousereplacement systems to enable the creation of accessible graphical user interfaces. To illustrate these techniques, they created Camera Canvas, an image editing application that uses a sliding toolbar as its universal menu controller. The parameters of the toolbar automatically adapt to the movement abilities of the specific user. During the experiments, the authors observed individuals with and without disabilities and of a variety of ages using Camera Canvas and found that the proposed techniques worked across many different movement abilities and experience levels. They also investigated how these techniques could be used to "retrofit" existing Windows applications with new graphical user interfaces and they created a tool called Menu Controller that can automatically re-render the menus of some existing applications into adaptive sliding toolbars.

The Wang et al.'s [9] paper is in the field of human emotion detection utilizing facial expressions, a field considered important in a variety of pervasive applications in 
assistive environments. The authors propose to place the emotion detection problem under the framework of discriminant Laplacian embedding (DLE) to integrate two types of facial expression data in a shared subspace, such that the advantages of both are exploited. Due to the reinforcement between the two types of facial features, the new data representation is more discriminative and easier to classify. Encouraging experimental results of empirical studies demonstrate the practical usage of the proposed DLE method for emotion detection.

Meza-Kubo et al. [10] in their paper present case studies to understand older adults' interactions in CS sessions and their relation with technologies and with members in their social family networks (SFN). To address the technological, social and illiteracy gaps, they designed and evaluated a pervasive CS collaborative system which eases interaction through natural interfaces and enables SFN members to interact with older adults during their CS activities regardless of their physical location. The evaluation results provide evidence that participants perceived the system as useful, easy to use and giving a pleasurable user experience. Furthermore, the results show the feasibility of augmenting traditional board games (e.g., chess, checkers) to create pervasive CS collaborative applications and the importance and benefits of integrating SFN members as informal caregivers.

Mehdi and Berns [11] focus on the development of a behavior-based strategy for an indoor autonomous mobile robot to locate an elderly person living alone in an unstructured home environment. In order to validate all aspects of the robotic behavior over a long period of time before using the robot in an elderly care environment, a simulated environment resembling an existing apartment and a simulated robot are presented. To increase the realism of the 3D simulation, an animated human character has been developed that walks to different rooms and exhibits different postures during its movements. The simulation maintains as a state the presence of the person in different rooms at different times of the day. Based on the perceived state, the estimation of possible locations of the person is carried out using a Markov decision process (MDP). The implemented methodology focuses mainly on two criteria for searching for the person: (a) the distance of the robot to the destination and (b) the likelihood of finding the person at that location. It also takes into account the last known position of the person for the final estimation of the possible location. The robot navigates, in simulation, autonomously to the desired location by planning a path and avoiding the obstacles in the way. The results from the $3 \mathrm{D}$ simulation show the effectiveness of the developed methodology.

Doukas et al. [12] deal with the emerging technologies of the future Internet (FI), which introduces new ways of networking and cognitive data delivery. In their paper, the potential of FI-based architectures for enabling the contextaware content adaptation and specialized delivery of health-related information in assistive environments is investigated. They present a system that utilizes the Publish/Subscribe Internetworking (PSI) architecture, an information-oriented architecture built for the FI using the so-called Publish/Subscribe paradigm. The approach uses information to enable flexibility, seamless information morphing and exploitation of context, access control and security. The paper also describes the implementation of an assistive environment that uses Blackadder, PSI's prototype, and illustrates its potential in the context of an emergency healthcare service scenario.

In the field of intelligent remote home monitoring, Maragoudakis and Loukis [13] propose a methodology for automatic real-time screening of heart sound signals (one of the most widely acquired signals from the human body for physiological as well as cognitive diagnostic purposes), to identify persons with abnormal heart condition who require some action to be taken. Their methodology is based on a novel Markov Chain Monte Carlo (MCMC) Bayesian Inference approach, which estimates conditional probability distributions in structures obtained from a treeaugmented naïve Bayes (TAN) algorithm. The specific method has been applied and validated in a highly "difficult" heterogeneous dataset of 198 heart sound signals, which comes from both healthy medical cases and unhealthy ones having aortic stenosis, mitral regurgitation, aortic regurgitation or mitral stenosis. The proposed methodology achieved high classification performance in this difficult screening problem. It performs higher than other widely used classifiers, showing great potential for contributing to a cost-effective large-scale application of computer-based assistive environment technologies.

In the paper by Diaz-Orueta et al. [14], the authors show how some cognitive functions (such as attention or verbal memory) may determine the interaction of cognitively impaired elderly people with technology. Twenty participants ranging from mild cognitive impairment to moderate Alzheimer's disease were assessed by means of a neuropsychological and functional battery and were asked to follow simple commands from an Avatar appearing on a TV by means of a remote control, such as asking the participant to confirm their presence or to respond with a "Yes" or "No" to a proposal to see a TV program. The number of correct answers and command repetitions required for the user to respond were registered. Results show that participants with a better cognitive and functional state in specific tests show a significantly better performance in the TV-decision task.

The paper by Reiss and Stricker [15] focuses on the monitoring of aerobic activities and targets two main goals: 
to estimate the intensity of activities and to identify basic/ recommended physical activities and postures. This relates to cognitive technologies, as physical exercise is an indicator of cognitive state. The author experimented using a dataset with 8 subjects, where 14 different activities were recorded, including the basic activities and postures, but also examples of household (ironing, vacuum cleaning), sports (playing soccer, rope jumping) and everyday activities (ascending and descending stairs). Data from three accelerometers and a heart rate monitor were analyzed. The paper presents the entire data processing chain, with analysis and comparison of different classification techniques, concerning also their feasibility for portable online activity monitoring applications. Satisfactory results are presented with various combinations of the sensors.

Finally, the paper by Karampiperis et al. [16] presents the event recognition designer toolkit (ERDT), a graphical authoring tool, with which a domain expert can design event recognition rules and produce standalone Event Recognizers. Their goal is to develop a user-friendly graphical tool with a basic set of operators, so that a user could easily produce recognizers for different domains and, when needed, easily extend the tool in order to satisfy domain-specific requirements. The ERDT uses an extendable pool of ER language libraries (at the moment SQL and Event Calculus are supported) and transforms the designed rules into Event Recognizers that use the preferred ER language. The same rule can be expressed in different languages without any changes to the design. Furthermore, the authoring tool is cross-platform, free and open source, so that it can be shared with the community, maximizing its potential impact in assistive environments.

In conclusion, this special issue presents novel data capture and processing technologies, which appear in different modalities. These technologies, in conjunction with almost unlimited bandwidth and storage, will enable the development of advanced cognitive systems that simulate the physical world and help improve the quality of life. While the intelligence of assistive environments of today is not yet complete, fully integrated or $100 \%$ accurate, the papers in this issue provide a sample to the challenges faced, the diversity of data and applications, and solutions that aim to achieve seamless assistive environments to support the user. As the world population is getting older, it needs such technological support to highlight and integrate these different solutions in order to provide human independence. This is the concept, which we, as guest editors, tried to highlight with the selection of the papers included in this special issue. From our position, we wish to thank the Editor-in-Chief of the International Journal Universal Access in the Information Society, Professor Constantine Stephanidis, for his constant support and help during the preparation of this issue and his exquisite editorial work.
We would also like to thank all the authors for their contributions and the reviewers for their assessment of the papers. We hope that the readers of the UAIS Journal will find the papers of this special issue interesting.

The Guest Editors

Ilias Maglogiannis, Fillia Makedon, Grammati Pantziou, Magrit Betke

\section{List of reviewers}

Vassilis Athitsos, University of Texas at Arlington, USA Pangiotis Bamidis, Aristotle University of Thessaloniki, Greece

Eric Becker, University of Texas at Arlington, USA

Petros Belsis, Technological Educational Institution of Athens, Greece

Gerard Bieber, Fraunhofer Institute, Germany

Alethea Blackler, Queensland University of Technology, Australia

Guanling Chen, University of Texas at Arlington, USA

Charalampos Doukas, University of the Aegean, Greece

Samuel Epstein, Boston University, USA

Dimitris Geneiatakis, University of Piraeus, Greece

Jef Guarante, Boston University, USA

Stathes Hadjieftymiades, University of Athens, Greece

Heng Huang, University of Texas at Arlington, USA

George Kambourakis, University of the Aegean, Greece

Dimitris Kosmopoulos, Rutgers University, USA

Vasso Koufi, University of Piraeus, Greece

Efthyvoulos Kyriacou, Frederik University, Cyprus

Christopher Kwan, Boston University, USA

Vangelis Metsis, University of Texas at Arlington, USA

Eric Missimer, Boston University, USA

Juha Pärkkä, VTT Technological Research Center, Finland

Isaac Paquette, Boston University, USA

Vasilis Plagianakos, University of Central Greece, Greece

Dimitris Vassis, Technological Educational Institution of

Athens, Greece

Dimitris Vergados, University of Piraeus, Greece

Zheng Wu, Boston University, USA

\section{References}

1. Maglogiannis, I., Hadjiefthymiades, S.: EmerLoc: location-based services for emergency medical incidents. Int. J. Med. Inform. 76(10), 747-759 (2007)

2. Doukas, C., Maglogiannis, I.: Adaptive transmission of medical image and video using scalable coding and context-aware wireless medical networks. EURASIP J Wireless Commun. Netw. Article ID 428397, pp. 12 (2008). doi:10.1155/2008/428397

3. Doukas, C., Metsis, V., Becker, E., Le, Z., Makedon, F., Maglogiannis, I.: Digital cities of the future: extending @ $@$ home 
assistive technologies for the elderly and the disabled. Telematics Inform. 28(3), 176-190 (2011)

4. Maglogiannis, I., Makedon, F., Pantziou, G.E., Baillie, L.: Pervasive technologies and assistive environments: social impact financial, government and privacy issues. Univers. Access Inf. Soc. 10(2), 111-114 (2011)

5. Maglogiannis, I.: Introducing intelligence in electronic healthcare systems: state of the art and future trends. AI Int. Perspect. Lecture Notes Artif. Intell. 5640, 71-90 (2009)

6. Doukas, C., Maglogiannis, I.: An assistive environment for improving human safety utilizing advanced sound and motion data classification. Univers. Access Inf. Soc. (UAIS) 10(2), 217-228 (2011)

7. Doukas, C., Maglogiannis, I.: Emergency fall incidents detection in assisted living environments utilizing motion, sound and visual perceptual components. IEEE Trans. Inf. Technol. Biomed. 15(2), 277-289 (2011)

8. Kwan, C.W., Paquette, I., Magee, J.J., Betke, M.: Adaptive sliding menubars make existing software more accessible to people with severe motion impairments (this issue). doi:10.1007/ s10209-013-0295-2

9. Wang, H., Huang, H., Makedon, F.: Emotion detection via discriminant Laplacian embedding (this issue). doi:10.1007/s10209013-0312-5
10. Meza-Kubo, V., Morán, A.L., Rodríguez, M.D.: Bridging the gap between illiterate older adults and cognitive stimulation technologies through pervasive computing (this issue). doi:10.1007/ s10209-013-0294-3

11. Mehdi, S.A., Berns, K.: Behavior based search of human by an autonomous indoor mobile robot in simulation (this issue). doi:10.1007/s10209-013-0301-8

12. Doukas, C., Fotiou, N., Polyzos, G.C., Maglogiannis, I.: Cognitive and context aware assistive environments using future internet technologies (this issue). doi:10.1007/s10209-013-0299-y

13. Maragoudakis, M., Loukis, E.: Heart sound screening in real-time assistive environments through MCMC Bayesian data mining (this issue). doi:10.1007/s10209-013-0293-4

14. Diaz-Orueta, U., Etxaniz, A., Gonzalez, M.F., Buiza, C., Urdaneta, E., Yanguas, J.: Role of cognitive and functional performance in the interactions between elderly people with cognitive decline and an avatar on TV (this issue). doi:10.1007/s10209-0130288-1

15. Reiss, A., Stricker, D.: Aerobic activity monitoring: towards a global approach (this issue). doi:10.1007/s10209-013-0292-5

16. Karampiperis, P., Mouchakis, G., Paliouras, G., Karkaletsis, V.: ER designer toolkit: a graphical event definition authoring tool (this issue). doi:10.1007/s10209-013-0300-9 